Perineuritis unassociated with other significant pathological changes is a rare finding. A patient is described with mild, non-insulin dependent diabe-

Editors tes mellitus who presented with the clinical picture of mononeuritis multiplex, and in whom perineuritis but no occlusive vascular disease was found

Robert C. Griggs, MD

Rochester, New York

Jerry R. Mendell, MD

Columbus, Ohio

Robert G. Miller, MD

San Francisco, California on sural nerve biopsy. Treatment with prednisone and plasma exchange resulted in some improvement. We conclude that the focal perineuritis, rather than the diabetes, was responsible for the clinical picture mimicking mononeuritis multiplex. In conjunction with previous reports, this suggests that perineuritis may be a treatable neuropathy.

Key words: perineuritis $\bullet$ neuropathy $\bullet$ mononeuritis multiplex $\bullet$ autoimmune disorders • plasma exchange

MUSCLE \& NERVE 15:630-635 1992

\title{
CASE-OF-THE-MONTH: PERINEURITIS PRESENTING AS MONONEURITIS MULTIPLEX
}

ZACHARY SIMMONS, MD, JAMES W. ALBERS, MD, PhD, and ANDERS A.F. SIMA, MD, PhD

Inflammatory involvement of the perineurium is a relatively common pathological finding that has been described in association with both sensory and sensorimotor neuropathy. ${ }^{3,5,6,14,15,18}$ It most frequently occurs as part of a process causing more widespread peripheral nerve involvement, and has been noted in cryoglobulinemia with vasculitis, ${ }^{14}$ sarcoidosis, ${ }^{16}$ leprosy, ${ }^{19}$ schistosomiasis, ${ }^{11}$ and ingestion of adulterated rapeseed oil. ${ }^{18}$ The occurrence of perineuritis in the absence of other significant pathological changes is rare. ${ }^{3,5,6,15}$ In this report, we describe a patient with perineuritis and mild diabetes mellitus presenting with the clinical picture of mononeuritis multiplex.

\section{CASE REPORT}

A 75-year-old woman with mild Parkinson's disease and mild non-insulin dependent diabetes mellitus suddently developed pain in her left hand, followed by weakness and atrophy. One month later, she noted left thigh pain and left leg weakness, followed by similar symptoms on the

From the Departments of Neurology (Drs. Simmons and Albers) and Pathology and Internal Medicine (Dr. Sima), University of Michigan, Ann Arbor, Michigan

Address reprint requests to James $W$. Albers, MD, PhD, Department of Neurology, University of Michigan Medical Center, 1500 East Medical Center Drive, Ann Arbor, Ml 48109.

Accepted for publication June 10, 1991

CCC 0148-639X/92/050630-06 \$04.00

(c) 1992 John Wiley \& Sons, Inc. right. The thigh pain resolved over 1 month, but she then noted calf pain, first on the left, then right. Her calf pain persisted, the leg weakness progressed, and by 4 months after the onset of symptoms she became unable to walk without assistance. She had been treated with a large number of medications in the year prior to presentation, including chlorodiazepoxide, chlorpromazine, hydrochlorothiazide, meprobamate, lasix, meclizine, propranolol, levadopa/carbidopa, digoxin, thyroid extract, glyburide, propoxyphene, acetaminophen, prazosin, diltiazem, amitriptyline, sublingual nitroglycerin, and iron, calcium, and potassium supplements.

When initially evaluated, approximately 6 months after the onset of symptoms, the patient had moderate atrophy and weakness of the left hand intrinsic muscles, with mild diffuse weakness of the other muscles of the left upper extremity. In the lower extremities, strength was graded as follows (Medical Research Council grades 0-5, right/left): hip flexors $2 / 3$, hamstrings $2 / 3$, quadriceps $3 / 4$, ankle dorsiflexors $0 / 0$, ankle plantar flexors 2/2. Reflexes were depressed in the left upper extremity and at both knees. Achilles reflexes were absent. Sensory examination revealed decreased pinprick and vibration distally in all extremities, worse in the legs than the arms, and worse in the left hand than the right. She could stand and walk only with assistance.

The following studies were normal or negative: complete blood count, routine blood chemis- 
try, serological test for syphilis, sedimentation rate, anti-nuclear antibody, rheumatoid factor, T4, T3, thyroid stimulating hormone, B 12, folic acid, creatine kinase, Borrelia burgdorferi antibodies, cryoglobulins, and urine arsenic, mercury, and lead. Serum protein electrophoresis revealed hypogammaglobulinemia. Her glycosylated hemoglobin and her fasting blood glucose while on an oral hypoglycemic agent were normal. Urine immunoelectrophoersis was negative for BenceJones proteins.

Myelogram and post-myelographic computerized tomographic scan of the lower thoracic and lumbosacral spines revealed only mild degenerative changes. Cerebrospinal fluid (CSF) examination was traumatic; there were $2270 \mathrm{RBC}$ per cubic millimeter and one WBC per cubic millimeter. CSF protein was $79 \mathrm{mg} / \mathrm{dL}$ and glucose was 68 $\mathrm{mg} / \mathrm{dL}$. A repeat lumbar puncture was nontraumatic and revealed only a mildly elevated protein level; cytology was negative.

The patient underwent 5 courses of therapeutic plasma exchange and was placed on prednisone $60 \mathrm{mg} /$ day. When seen two months after the initiation of treatment, her pain had resolved. Her neurological examination showed a mild in- crease in the strength of proximal lower extremity muscles.

\section{MATERIALS AND METHODS}

Motor and sensory nerve conduction studies, including $\mathrm{F}$-wave recordings, were performed using standard techniques of supramaximal percutaneous stimulation, recording from surface electrodes. Skin temperature was maintained at $32^{\circ} \mathrm{C}$ or greater. Electromyography was performed using standard concentric needles.

A left sural nerve biopsy was obtained. One portion of the nerve was formalin fixed and paraffin embedded. Sections were stained with hematoxylin \& eosin, Gomori's trichrome, and peroxidase-antiperoxidase diluted in tris buffer for IgG (dilution 1/200), $\operatorname{IgM}(1 / 100)$, and $\operatorname{IgA}(1 / 200)$. Control stains were performed using rabbit serum. A second portion of the biopsy was snapfrozen and used for immunofluorescence staining for IgG, IgM, and C3. A third portion of the biopsy was fixed in $2 \%$ glutaraldehyde in phosphate buffer ( $\mathrm{pH} 7.2$ ), postfixed in $1 \%$ osmic acid, dehydrated, and embedded in epoxy resin. Ultrathin sections were examined ultrastructurally.

Table 1. Nerve conduction studies before and two months after treatment of perineuritis with prednisone and plasma exchange

\begin{tabular}{|c|c|c|c|c|c|c|c|c|c|}
\hline \multirow[t]{2}{*}{ Nerve } & \multicolumn{3}{|c|}{ Amplitude (mv or $\mu v$ ) } & \multicolumn{3}{|c|}{ Latency (msec) } & \multicolumn{3}{|c|}{ Conduction velocity $(\mathrm{m} / \mathrm{sec})$} \\
\hline & $\mathrm{R}$ & $L$ & Norm & $R$ & L & Norm & $\mathrm{R}$ & $\mathrm{L}$ & Norm \\
\hline \multicolumn{10}{|l|}{ Pretreatment } \\
\hline \multicolumn{10}{|l|}{ Sensory } \\
\hline Median & 18 & NR & $>20$ & 4.3 & & $<3.7$ & & & \\
\hline Uinar & 6 & NR & $>10$ & 3.9 & & $<3.5$ & & & \\
\hline Sural & NR & NE & $>6$ & & & $<4.2$ & & & \\
\hline \multicolumn{10}{|l|}{ Motor } \\
\hline Median & 13.8 & 2.0 & $>4$ & 4.0 & 5.8 & $<4.4$ & 53 & 31 & $>49$ \\
\hline Ulnar & 12.2 & 1.7 & $>6$ & 3.5 & 5.1 & $<3.5$ & 55 & 39 & $>49$ \\
\hline Peroneal & NR & NE & $>2$ & & & $<6.1$ & & & $>41$ \\
\hline Peroneal-AT & 0.1 & NE & & 3.5 & & & & & \\
\hline Tibial & $\mathrm{NE}$ & $\mathrm{NE}$ & $>3$ & & & $<6.1$ & & & $>41$ \\
\hline Ulnar F-wave & & & & 27.8 & & $<31$ & & & \\
\hline \multicolumn{10}{|l|}{ Posttreatment } \\
\hline \multicolumn{10}{|l|}{ Sensory } \\
\hline Median & 22 & 3 & $>20$ & 4.2 & 5.0 & $<3.7$ & & & \\
\hline UInar & 16 & NR & $>10$ & 3.8 & & $<3.5$ & & & \\
\hline Sural & NR & $\mathrm{NE}$ & $>6$ & & & $<4.2$ & & & \\
\hline \multicolumn{10}{|l|}{ Motor } \\
\hline Median & 9.5 & NR & $>4$ & 3.4 & & $<4.4$ & 41 & & $>49$ \\
\hline Ulnar & 8.6 & 1.9 & $>6$ & 3.2 & 4.1 & $<3.5$ & 47 & 37 & $>49$ \\
\hline Peroneal & $\mathrm{NE}$ & $\mathrm{NE}$ & $>2$ & & & $<6.1$ & & & $>41$ \\
\hline Peroneal-AT & 0.3 & NR & & 5.9 & & & & & \\
\hline Tibial & 1.4 & 0.7 & $>3$ & 5.4 & 6.8 & $<6.1$ & & & $>41$ \\
\hline Ulnar F-wave & & & & 28.6 & & $<31$ & & & \\
\hline
\end{tabular}

$N R=$ no response; $N E=$ not examined; $A T=$ anterior tibialis recording. 


\section{RESULTS}

Nerve conduction study results are shown in Table 1. Upon initial evaluation, there was marked asymmetry of median and ulnar action potential amplitudes. Lower extremity responses were small or absent. No features of abnormal temporal dispersion or partial conduction block were noted on proximal stimulation. Needle examination of the lower extremities revealed increased insertional activity and a marked degree of abnormal spontaneous activity in proximal and distal muscles bilaterally, including lumbar paraspinal muscles. Voluntary motor unit potentials were either absent or were large and polyphasic with decreased recruitment. In the upper extremities, asymmetry was noted, with prominent abnormal spontaneous activity in left median and ulnar-innervated muscles. The cervical paraspinal muscles were spared. Affected muscles exhibited large, polyphasic voluntary motor unit action potentials with decreased recruitment.

Two months after the initiation of treatment, sensory nerve conduction studies were mildly improved. Needle examination revealed decreased spontaneous activity and increased recruitment in some lower extremity muscles when compared with the prior study.

Light-microscopic examination of the sural nerve revealed several fascicles with focal thickening of the perineurium (Fig. 1). The individual laminae were spread apart by collagen and inflammatory cells consisting mainly of lymphocytes and histiocytes. The myelinated fiber density was decreased adjacent to involved areas of the perineurium, and the endoneurium appeared edematous in these areas (Fig. 2). Arterioles in the epineurium showed occasional perivascular lymphocyte cuffing. The vessel walls were unaffected.

Immunoperoxidase-stained sections showed diffuse leakage of $\operatorname{IgG}, \operatorname{IgM}$, and IgA into the endoneurial space through affected areas of the perineurium (Fig. 3). Control incubations using rabbit serum were negative. Immunofluorescence-stained sections revealed nonspecific leakage of $\operatorname{IgG}, \operatorname{IgM}$, and C3 through affected parts of the perineurium. Electron-microscopic examination showed splitting and discontinuities of the perineurial laminae with increased deposition of collagen (Fig. 4). In close proximity to affected parts of the perineurium, occasional myelinated fibers undergoing Wallerian degeneration were seen. In the same areas, endoneurial collagen appeared to be increased.

\section{DIscussion}

This report describes a patient whose clinical examination is most suggestive of mononeuritis mul-

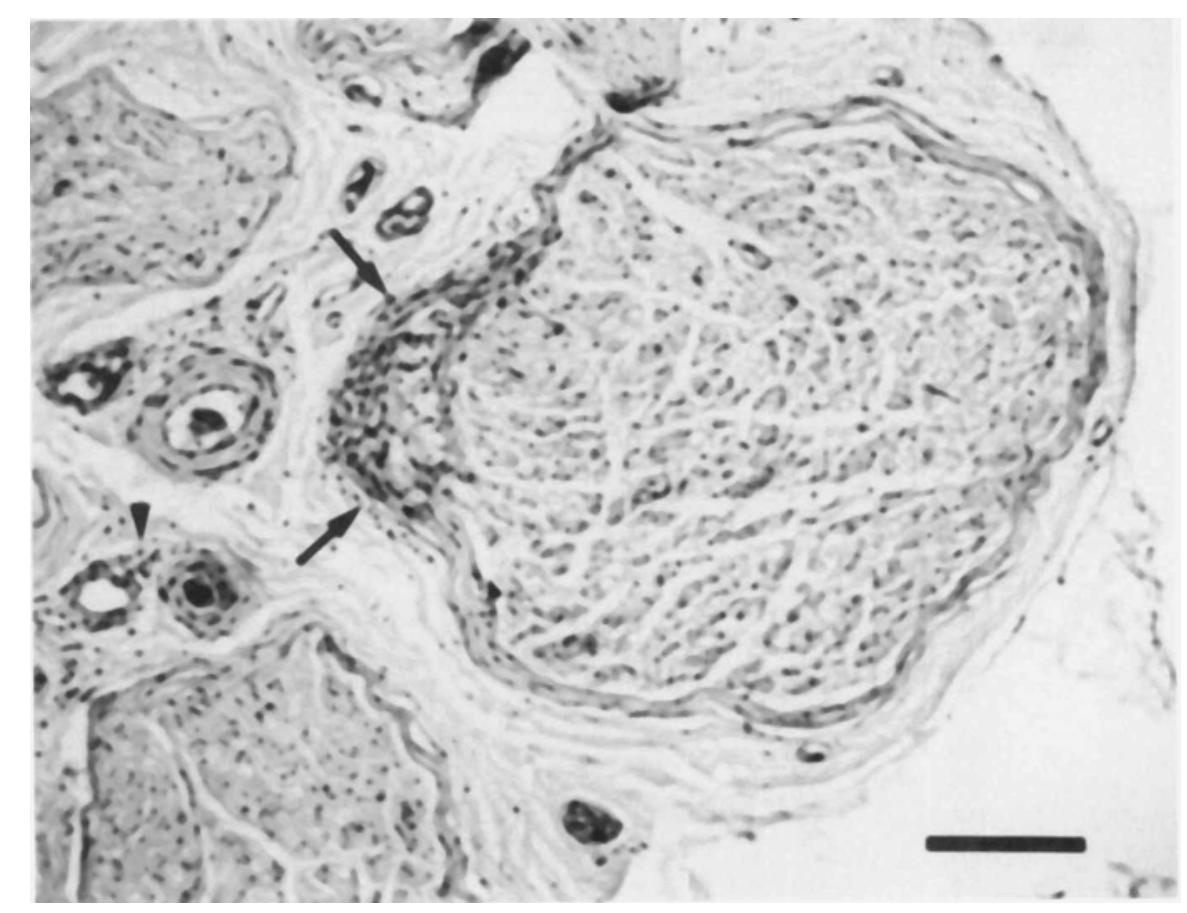

FIGURE 1. Hematoxylin \& eosin-stained section showing focal perineuritis (arrows) and discrete perivascular lymphocyte infiltration in the epineurium (arrowhead). Bar $=200 \mu \mathrm{m}$. 


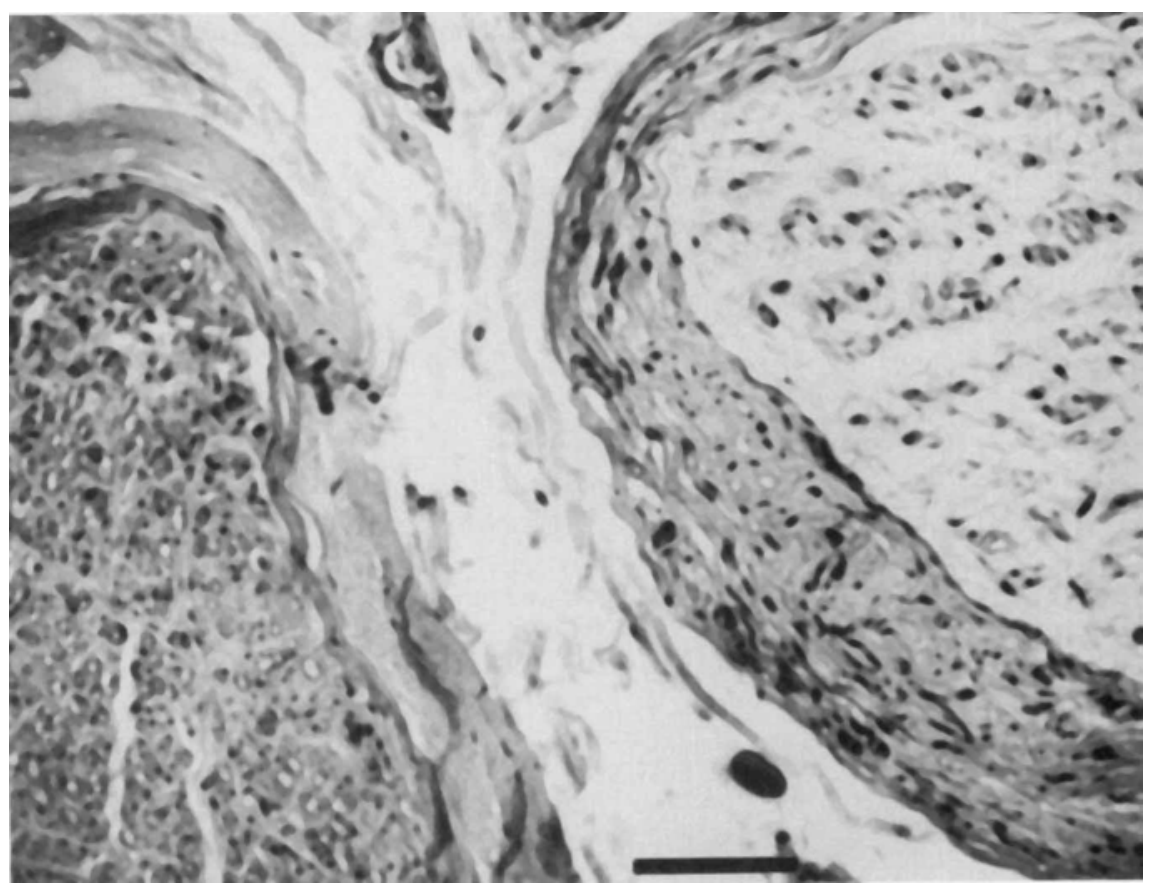

FIGURE 2. Focal perineuritis with marked thickening of the perineurium secondary to collagen deposition (right). The affected fascicle shows loss of myelinated fibers and endoneurial edema compared with the unaffected fascicle on the left. Hematoxylin \& eosin; Bar $=50 \mu \mathrm{m}$.

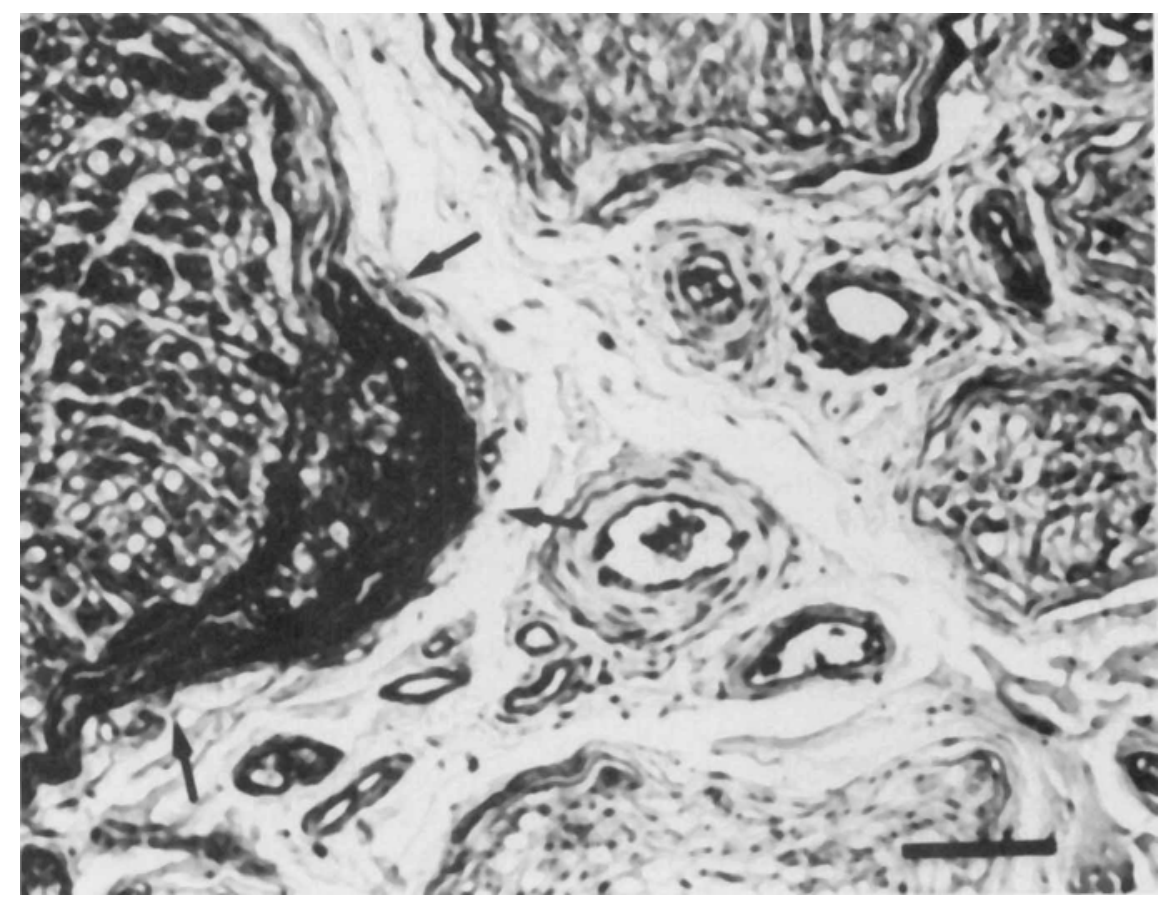

FIGURE 3. Immunoperoxidase stained section incubated for IgM. The affected perineurium shows deposition of $\operatorname{lgM}$ (arrows) with leakage into the endoneurial space. Bar $=50 \mu \mathrm{m}$. 


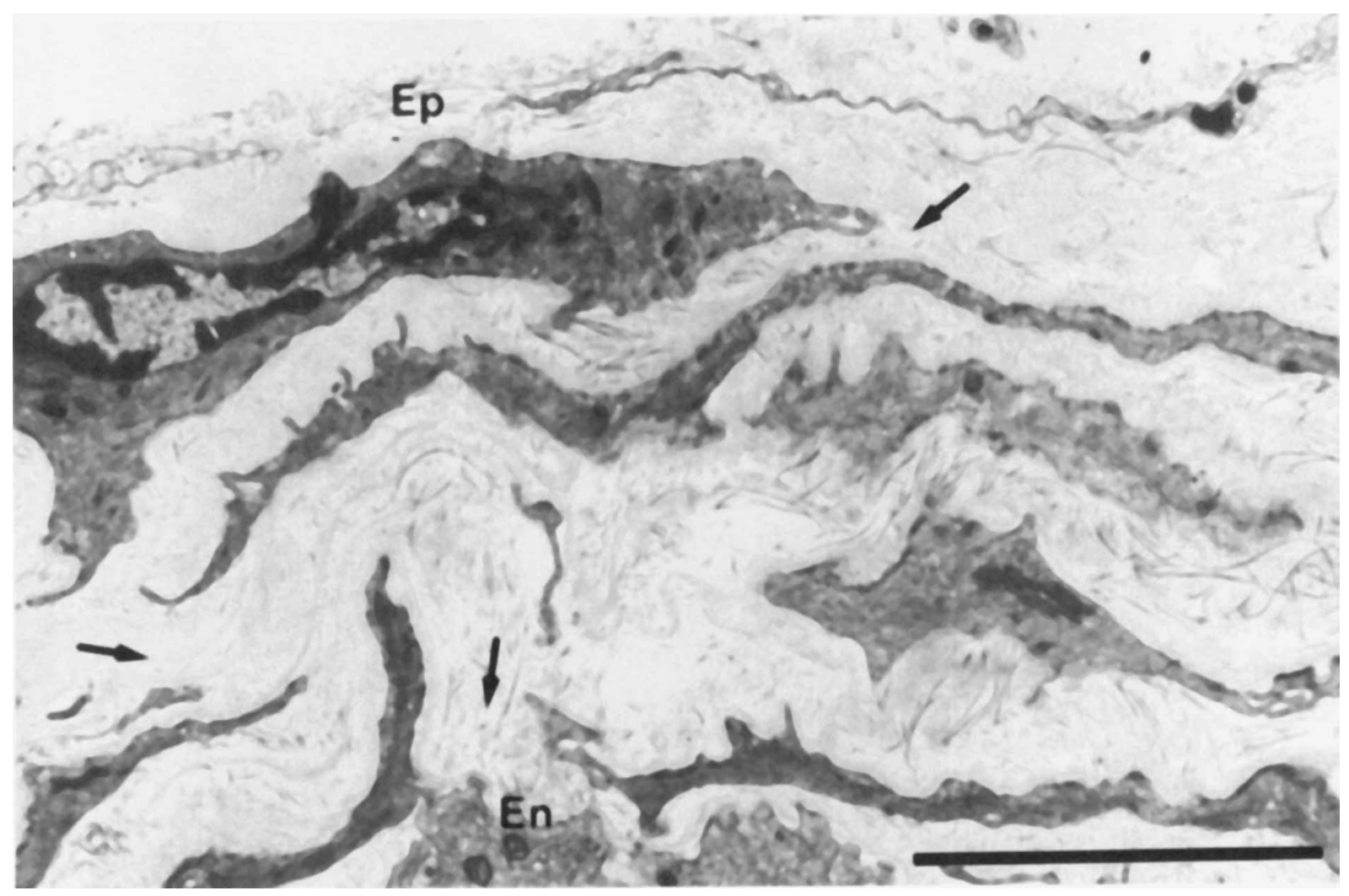

FiGURE 4. Electron micrograph showing splitting and discontinuities of the perineurial laminae (arrows). Ep: epineurial side; En: endoneurial side; $\operatorname{Bar}=5 \mu \mathrm{m}$.

tiplex. Despite very mild diabetes characterized by normal fasting blood glucose and glycosylated hemoglobin while on small doses of an oral hypoglycemic agent, the patient sustained a rapidly progressive and debilitating course secondary to her peripheral neuropathy. This raised the possibility of other etiologies for the neuropathy, particularly a necrotizing peripheral nervous system vasculitis, and led to sural nerve biopsy. Examination of the biopsy revealed focal perineuritis as the significant pathological feature.

Although thickening of the perineurial basement membrane has been described in association with diabetes, ${ }^{2,13}$ inflammatory involvement of the perineurium is not regarded as a feature of diabetic neuropathy, suggesting that the perineuritis in this patient occurred independently of the mild diabetes and may have been the cause of the asymmetrical peripheral neuropathy. We cannot completely exclude the possibility that the patient's neuropathy was secondary to microvascular disease. However, the absence of ischemic endoneurial changes and/or nerve fiber regeneration in the biopsy would argue against this and in favor of perineuritis as the causal factor. Though sensorimotor mononeuropathy multiplex may be due to a wide variety of other underlying causes, including collagen vascular disorders, infection, sarcoidosis, malignancy, acquired demyelination, and compression, ${ }^{12,17}$ none of these was present in our patient. 'To this list, we propose adding perineuritis.

Perineuritis as a unique clinicopathological entity was first described by Asbury et al. ${ }^{3}$ Their two patients developed a predominantly sensory mononeuropathy multiplex, with variable, incomplete resolution. Three subsequent reports of perineuritis with nerve biopsy findings virtually identical to ours have appeared. Clinical presentations have varied considerably, from patchy sensory loss and dysesthesias, ${ }^{15}$ to a sensoriomotor polyneuropathy, ${ }^{5}$ to a clinical and electrodiagnostic picture resembling chronic inflammatory demyelinating polyneuropathy. ${ }^{6}$

Though the pathogenesis of perineuritis remains obscure, several pieces of evidence point toward an immune-mediated mechanism. These include immunoperoxidase and immunofluor- 
escence studies ${ }^{5,6}$ as well as improvement with prednisone therapy. ${ }^{5,6,15}$ In addition, patients have been described in whom perineuritis is associated with ulcerative colitis ${ }^{6}$ and carcinoma, ${ }^{3,15}$ both of which may be associated with immune-mediated phenomena. ${ }^{4,8,10}$

A trial of therapeutic plasma exchange was undertaken in view of the efficacy of this procedure in other immune-mediated peripheral nervous system disorders including acute ${ }^{20}$ and chronic ${ }^{7,9}$ inflammatory demyelinating polyradiculoneuropathies. In 2 months, our patient, whose symptoms were progressing up to the time of treatment, had a substantial decrease in pain, some improvement in strength, and a decrease in spontaneous activity with an increase in recruitment on electromyography. All of these parameters suggest improvement, though the relationship between treatment and improvement remains speculative because the natural history of perineuritis is not known and spontaneous improvement may occur in patients with diabetic mononeuritis multiplex. Although nerve conduction results did not improve significantly from those obtained prior to treatment, this was not unexpected, since such improvement of ten lags behind clinical improvement. ${ }^{1}$ In addi- tion, the decreases in amplitude and conduction velocity in some motor nerves likely reflect disuse.

The results of the immunofluorescence and immunoperoxidase studies in our patient are nonspecific and suggest a breakdown of the perineurial diffusion barrier. Borque et al. ${ }^{5}$ suggested that the mechanism of nerve fiber damage in perineuritis may be increased permeability of the perineurium, with subsequent alteration of the endoneurial microenvironment. Our studies support this suggestion.

We cannot exclude the possibility of a toxic etiology of this patient's perineuritis, given the large number of medications used during the preceding year. The pathological findings reported here, however, are more limited in scope than those seen with the toxic perineuritis caused by adultered rapeseed oil. ${ }^{18}$

Perineuritis thus remains a poorly understood phenomenon. It appears to represent a rare but distinct pathological finding. Sural nerve biopsy should be considered in diabetic patients with an atypical presentation, since prednisone and plasma exchange may represent effective therapies.

\section{REFERENCES}

1. Albers JW: Inflammatory demyelinating polyradiculoneuropathy. in Brown WF, Bolton CF (eds). Clinical Electromyography. Boston, Butterworths, 1987:209-244.

2. Asbury AK, Johnson PC: Pathology of Peripheral Nerve. Philadelphia, WB Saunders, 1978:99.

3. Asbury AK, Picard EH, Baringer JR: Sensory perineuritis. Arch Neurol 1972;26:302-312.

4. Bayless TM: Ulcerative colitis. in Gitnick G (ed). Principles and Practice of Gastroenterology and Hepatology. New York, Elsevier, 1988:461-496.

5. Borque CN, Anderson BA, del Campo CM, Sima AAF: Sensorimotor perineuritis - an autoimmune disease? Can J Neurol Sci 1985;12:129-133.

6. Chad DA, Smith TW, DeGirolami U, Hammer K: Perineuritis and ulcerative colitis. Neurology 1986;36:1377-1379.

7. Donofrio PD, Tandan R, Albers JW: Plasma exchange in chronic inflammatory demyelinating polyradiculoneuropathy, Muscle Nerve 1985;8:321-327.

8. Dropcho $E$ J: The remote effects of cancer on the nervous system. Neurol Clin 1989;7:579-603.

9. Dyck PJ, Daube J, O'Brien P, et al: Plasma exchange in chronic inflammatory demyelinating polyradiculoneuropathy. N Engl J Med 1986;314:461-465.

10. Fielding JF: Clinical features of ulnerative colitis. in Misiewicz JJ, Pounder RE, Venables CW (eds). Diseases of the Gut and Pancreas. Oxford, Blackwell Scientific Publications, 1987:725-744
11. Ghoneim MA, Ashamallah A, El-Bolkainy MN: Role of perineuritis in the atonic dilated bilharzial ureter. Int Surg 1976;61:411-412

12. Hellman DB, Laing TJ, Petri M, Whiting-O'Keefe Q, Parry GJ: Mononeuritis multiplex: The yield of evaluations for occult rheumatic diseases. Medicine 1988;67:145-153.

13. Johnson PC, Brendel K, Meezan E: Human diabetic perineurial cell basement membrane thickening, Lab Invest $1981 ; 44: 265-270$.

14. Konishi T, Saida K, Ohnishi A, Nishitani H: Perineuritis in mononeuritis multiplex with cryoglobulinemia. Muscle Nerve 1982;5:173-177

15. Matthews WB, Squier MV: Sensory perineuritis. I Neurol Neurosurg Psychiatry 1988;51:473-475.

16. Oh SJ: Sarcoid polyneuropathy: A histologically proved case. Ann Neurol 1980;7:178-181.

17. Parry GJG: Mononeuropathy multiplex (AAEE case report \# 11). Muscle Nerve. 1985;8:493-498.

18. Ricoy JR, Cabello A, Rodriguez J, Tellez I: Neuropathological studies on the toxic syndrome related to adulterated rapeseed oil in Spain. Brain 1983;106:817-835.

19. Sabin TD, Swift TR: Leprosy. in Dyck PJ, Thomas PK, Lambert EH, Bunge R (eds) Peripheral Neuropathy (ed 2). Philadelphia, WB Saunders, 1984:1955-1987.

20. The Guillain-Barre Study Group. Plasmapheresis and acute Guillain-Barre syndrome. Neurology 1985;35:10961104. 